\title{
Projective Geometry LDPC Codes for Ultralong-Haul WDM High-Speed Transmission
}

\author{
Ivan B. Djordjevic and Bane Vasic, Senior Member, IEEE
}

\begin{abstract}
In this letter, we investigate the performance of low-density parity-check (LDPC) codes in long-haul optical communication systems for carrier-suppressed return-to-zero signal format at $40 \mathrm{~Gb} / \mathrm{s}$ per channel in a wavelength-division-multiplexing environment. We are particularly concerned with high-rate codes based on projective geometries. These codes have large minimum distance and simple iterative decoding algorithms, which makes them good candidates for such high-speed application. We consider iterative decoding based on min-sum algorithm since it requires only simple addition and minimum operations and, as such, is suitable for high-speed optical transmission. Contrary to the common practice of considering the performance of error controlling schemes using the additive white Gaussian noise channel assumption, we consider the performance of the proposed LDPC schemes, taking into account in a natural way all major impairments in long-haul optical transmission such as amplifier spontaneous emission noise, pulse distortion due to fiber nonlinearities, chromatic dispersion or polarization dispersion, crosstalk effects, intersymbol interference, etc.
\end{abstract}

Index Terms-Error control, iterative decoding, long-haul transmission, low-density parity-check (LDPC) codes, optical communications, projective geometries.

\section{INTRODUCTION}

$\mathbf{R}$ ECENTLY, a number of high-speed long-haul optical communications systems have been demonstrated, commercialized, or implemented [1]. Although data flow through these systems has increased tremendously, it has become widely recognized that full utilization of the available bandwidth cannot be achieved without powerful error-control schemes.

A significant effort has been made to apply error-control techniques to various optical transmission systems [2]-[5] with a special emphasis on turbo codes [6]. In a series of articles [7]-[9], we showed that error performance and decoder hardware complexity offered by turbo codes can be greatly improved by using other types of iteratively decodable coding schemes, in particular low-density parity check (LDPC) codes. In this letter, we are concerned with LDPC codes based on finite geometries [10] because they require only simple encoders and we can be assured of large minimum codeword distances. The structure of finite geometry codes is of crucial importance for high-speed implementations for two reasons: 1) These codes

Manuscript received December 17, 2002; revised January 31, 2003.

I. B. Djordjevic is with the Department of Electrical and Electronic Engineering, University of Bristol, Bristol BS8 1TR, U.K. (e-mail: Ivan.Djordjevic@bristol.ac.uk).

B. Vasic is with the Department of Electrical and Computer Engineering, University of Arizona, Tucson, AZ 85721, USA (e-mail: vasic@ece.arizona.edu).

Digital Object Identifier 10.1109/LPT.2003.810250 can lend themselves to encoders that can be realized by shift registers, and 2) due to their combinatorial structure, they can be decoded using reduced complexity belief-propagation algorithm.

Projective geometry based LDPC codes can be decoded by various belief-propagation decoding methods [10] such as one-step majority-logic decoding, bit flipping, and iterative decoding based on the sum-product algorithm. Although sum-product iterative decoding has been demonstrated to perform well in various types of channels, it is computationally intensive and it is not clear if it is suitable for optical communications at data rate $40 \mathrm{~Gb} / \mathrm{s}$ or higher. However, the min-sum version of this algorithm, which is an approximation of $a$ posteriori probability decoding, requires only simple addition and "finding minimum" operations and, as such, is suitable for high-speed optical transmission.

In order to assess the performance of the proposed coding scheme, we use a very realistic simulation model that takes into account in a natural way all major impairments in long-haul optical transmission such as amplifier spontaneous emission (ASE) noise, pulse distortion due to fiber nonlinearities, chromatic dispersion, crosstalk effects, intersymbol interference (ISI), etc. As noted in [7], this approach gives a much better picture of the code performance than the commonly used additive white Gaussian noise (AWGN) channel noise model [3]-[5]. Our extensive simulations show that the LPDC codes perform well in the presence of all these impairments, and we are confident in proposing their use in optical communications for high-speed long-haul transmission.

\section{SySTEM Model Description}

In wavelength-division-multiplexing (WDM) systems, multiple optical carriers at different wavelengths are modulated by using independent electrical signals and then transmitted over the same fiber. The optical signal at the receiver is split into separate channels by using an optical demultiplexer. Erbium-doped fiber amplifiers (EDFAs) and dispersion compensating fibers (DCFs) are deployed periodically to compensate the loss and accumulated dispersion of the standard single-mode fiber (SMF). A carrier-suppressed return-to-zero (CSRZ) modulator, considered here, is composed of a laser diode, a Mach-Zehnder (MZ) intensity modulator, another MZ modulator driven by a sinusoid at half bit-rate frequency, and an encoder. A typical direct detection receiver is composed of a polarization filter, an optical filter, a p-i-n photodiode, an electrical filter, a sampler, and a decision circuit, followed by a decoder. 
The propagation of a signal through the transmission media is modeled by the nonlinear Schrödinger equation [11]

$$
\begin{aligned}
\frac{\partial A}{\partial z}=-\frac{\alpha}{2}-\frac{i}{2} \beta_{2} \frac{\partial^{2} A}{\partial T^{2}}+ & \frac{\beta_{3}}{6} \frac{\partial^{3} A}{\partial T^{3}} \\
& +i \gamma\left(|A|^{2}-T_{R} \frac{\partial|A|^{2}}{\partial T}\right) A
\end{aligned}
$$

where $z$ is the distance propagated along the fiber, relative time $T=t-z / v_{g}$ gives a frame of reference moving at the group velocity $v_{g}, A(z, T)$ is the complex field amplitude of the pulse, $\alpha$ is the attenuation coefficient of the fiber, $\beta_{2}$ is the group velocity dispersion (GVD) coefficient, $\beta_{3}$ is the second-order GVD, $\gamma$ is the nonlinearity coefficient giving rise to Kerr effect nonlinearities, and $T_{R}$ is the Raman coefficient. Equation (1) can be solved using the split-step Fourier method, as described in [11].

The electrical field coming through the fiber to the optical filter input can be written as

$$
r(t)=s(t)+n(t), \quad s(t)=\sum_{n=-\infty}^{\infty} \sqrt{b_{n} P} p_{n}\left(t-n T_{b}\right)
$$

where $s(t)$ is the (optical) amplifier chain output signal field. In (2), $p_{n}(t)$ is the $n$th bit pulse shape, $P$ is the peak power, and $b_{n}$ is the (coded) information content $b_{n} \in\{r, 1\}$, with $r$ being the extinction ratio, $0 \leq r<1$. Both the ASE noise components and the multipath interference (MPI) components are combined into a noise process $n(t)$, which is considered to be colored Gaussian with autocorrelation function given by

$$
R_{n}(\tau)=R_{\mathrm{ASE}}(\tau)+R_{\mathrm{MPI}}(\tau) .
$$

[ $R_{\mathrm{ASE}}(\tau)$ and $R_{\mathrm{MPI}}(\tau)$ are $\mathrm{ASE}$ and MPI autocorrelation functions, respectively]. The power spectral density of ASE noise is determined by the EDFA output filter, while the spectrum of MPI noise is determined by the signal spectrum. The autocorrelation function of ASE noise is $R_{\mathrm{ASE}}(\tau)=N_{0} R_{\mathrm{EDFA}}(\tau)$, where $R_{\mathrm{EDFA}}(\tau)$ is the EDFA output optical filter autocorrelation function, and $N_{0}$ is the power spectral density of ASE noise in one state of polarization. Within the bandwidth of an optical filter in EDFA, the noise power spectral density function can be approximated as $N_{0} \approx n_{\mathrm{sp}}(G-1) h f N_{\mathrm{amp}}$, wherein $n_{\mathrm{sp}}$ is the spontaneous emission factor, $G$ is the EDFA gain, $h f$ is the photon energy, and $N_{\mathrm{amp}}$ is the number of amplifiers. $r(t), s(t)$, and $p_{n}(t)$ are in fact the complex envelopes of corresponding analytical signals.

The photodiode output noise process has the mean

$$
\overline{I(t)}=\int_{-\infty}^{\infty}|S(\tau)|^{2} h_{2}(t-\tau) d \tau+R_{N}(0)
$$

and the variance

$$
\begin{aligned}
\sigma^{2}(t)=2 R_{e}\left\{\int_{-\infty}^{\infty} \int_{-\infty}^{\infty} S(\tau) S^{*}(\hat{\tau}) R_{N}(\tau-\hat{\tau}) h_{2}(t-\tau)\right. \\
\left.\cdot h_{2}(t-\hat{\tau}) d \tau d \hat{\tau}\right\}+2 \int_{-\infty}^{\infty} \int_{-\infty}^{\infty} h_{2}(t-\tau) \\
\cdot\left|R_{N}(\tau-\hat{\tau})\right|^{2} h_{2}(t-\tau) d \tau d \hat{\tau}+q \overline{I(t)}+\sigma_{e}^{2}
\end{aligned}
$$

where $h_{1}(t)$ and $h_{2}(t)$ denote the impulse responses of the optical and electrical filter, respectively, and $S(t)$ is the optical filter output signal. The autocorrelation function of the optical filter output noise can be expressed as

$$
R_{N}(\tau)=\int_{-\infty}^{\infty} R_{h_{1}}(t) R_{n}(\tau-t) d t
$$

where $R_{h_{1}}(\tau)$ is the optical filter autocorrelation function. Notice that the photodiode output noise process is not stationary (the mean values and the standard deviation are functions of time). In (5), $\sigma_{e}^{2}$ is the electronic noise variance, which includes both transmitter and receiver electronic noise (also known as "back-to-back" noise), while $q \bar{I}$ is the photodiode shot noise variance ( $q$ is an electron charge). If the polarization filter is omitted, the second term in (5) should be multiplied by a factor of two. The derivation (4) and (5) is given in our previous paper [12].

\section{CODE CONSTRUCTION}

As mentioned in the introduction, our code construction is based on projective geometry. The idea of using projective geometry for code construction is not new but has attracted much attention lately, since it has been realized that some projective geometry codes are LDPC codes [10], [14]. The renewed interest for such codes is also due to the fact that they perform well when decoded by suboptimal iterative algorithms that have been developed recently [7], [8], [14].

In this section, we summarize basic structure and properties of projective geometry codes. The detailed description can be found in [10]. Note that the construction proposed here is more general than the one given in [10], since it employs the ground Galois field $[\mathrm{GF}(p)]$, of arbitrary (prime power) order $p$, whereas in [10] only the case $p=2$ was considered.

The finite projective geometries $\left[\mathrm{PG}\left(m, p^{s}\right)\right]$ are constructed using $(m+1)$-tuples $\mathbf{x}=\left(x_{0}, x_{1}, \ldots, x_{m}\right)$ of elements $x_{i}$ from $\mathrm{GF}\left(p^{s}\right)$ ( $p$-a prime, $s$-positive integer), not all simultaneously equal to zero, called points. Two $(m+1)$-tuples $\mathbf{x}=$ $\left(x_{0}, x_{1}, \ldots, x_{m}\right)$ and $\mathbf{y}=\left(y_{0}, y_{1}, \ldots, y_{m}\right)$ represent the same point if $\mathbf{y}=\lambda \mathbf{x}$, where $\lambda \in \mathrm{GF}\left(p^{s}\right)$ is a nonzero element. So each point can be represented on $p^{s}-1$ ways, referred to as the equivalence class. It is obvious than that the number of points in $\mathrm{PG}\left(m, p^{s}\right)$ is $v=\left[p^{(m+1) s}-1\right] /\left(p^{s}-1\right)$.

The equivalence classes (points) can be represented by $\left[\alpha^{i}\right]=\left\{\alpha^{i}, \beta \alpha^{i}, \beta^{2} \alpha^{i}, \ldots, \beta^{p^{s}-2} \alpha^{i}\right\},(0 \leq i \leq v)$, where $\beta=\alpha^{v}$. Let $\left[\alpha^{i}\right]$ and $\left[\alpha^{j}\right]$ be two distinct points in $\mathrm{PG}(m$, $\left.p^{s}\right)$. Then the line passing through them consists of points of the form $\left[\lambda_{1} \alpha^{i}+\lambda_{2} \alpha^{j}\right]$, where $\lambda_{1}, \lambda_{2} \in G F\left(p^{s}\right)$. Since $\left[\lambda_{1} \alpha^{i}+\lambda_{2} \alpha^{j}\right]$ and $\left[\beta^{k} \lambda_{1} \alpha^{i}+\beta^{k} \lambda_{2} \alpha^{j}\right]$ are the same point, each line in $\mathrm{PG}\left(m, p^{s}\right)$ consists of $k=$ $\left(p^{m s}-1\right) /\left(p^{s}-1\right)$ points. The number of lines intersecting at a given point is $\left(p^{m s}-1\right) /\left(p^{s}-1\right)$, and the number of lines in projective geometry is $b=\left(p^{s(m+1)}-1\right)\left(p^{s m}-1\right) /\left[\left(p^{2 s}-1\right)\left(p^{s}-1\right)\right]$.

Let us now form the incidence matrix $A=\left(a_{i j}\right)_{b x \nu}$ whose rows correspond to the lines and columns to the points, with columns being arranged in the order $\left[\alpha^{0}\right],\left[\alpha^{1}\right], \ldots,\left[\alpha^{v}\right] . a_{i j}=$ 1 if $j$ th point belongs to $i$ th line, and zero otherwise. Each row has weight $p^{s}+1$ and each column $\left(p^{m s}-1\right) /\left(p^{s}-1\right)$. Any two columns have exactly one " 1 " in common and any two rows at most one " 1 " in common. Therefore, the point-line incident matrix is in fact the matrix of parity checks $H$ of the LDPC code 

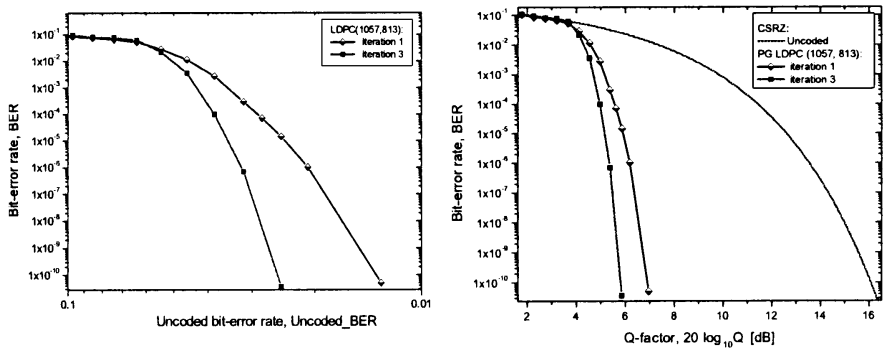

Fig. 1. BER performance of PG LDPC(1 057813$)$ code at $40 \mathrm{~Gb} / \mathrm{s}$.

and the corresponding Tanner graph is free of cycles of girth (the length of shortest cycle) 4 .

\section{PG LDPC CODE PERFormance}

In this section, we present the performance of LDPC codes in the presence of fiber nonlinearities, residual dispersion, ISI, and receiver noise resulting from signal-noise and noise-noise interaction in the $\mathrm{p}-\mathrm{i}-\mathrm{n}$ photodiode. The influence of the transfer functions of the optical and electrical filters is taken into account as well. A WDM system with $40-\mathrm{Gb} / \mathrm{s}$ bit rate per channel and a channel spacing of $100 \mathrm{GHz}$ is considered. It is assumed that the observed channel is located at $1552.524 \mathrm{~nm}(193.1 \mathrm{THz})$ and that there exists a nonnegligible interaction with six neighboring channels. The extinction ratio is set to $13 \mathrm{~dB}$. The transmitter and receiver imperfection is described through a back-to-back $Q$ factor, which is set to $23 \mathrm{~dB}$.

A projective geometry-based LDPC $(1057,813)$ code with code rate of $R=0.769$ (redundancy of $\approx 30 \%$ ), constructed as described in Section III for $m=p=2$ and $s=5(v=$ 1057), is considered as an illustration. Notice that the same code was discussed in [14], in the context of transmission of the binary phase-shift keying modulated signal over AWGN channel. However, none of the decoding algorithms presented in [14] is suitable for high-speed optical transmission since all of them require the knowledge of standard deviation of the noise samples. In an optical communication channel, the noise statistics are more complicated, which motivates the use of min-sum algorithm that requires the knowledge of received samples only. Another reason why min-sum algorithm is suitable for high-speed optical transmission is that it requires just addition and "finding minimum" operations.

Fig. 1 shows the bit-error-rate (BER) results of a Monte Carlo simulation. The optical filter is modeled as a super-Gaussian filter of order eight and bandwidth $1.5 \mathrm{R}_{b}\left(\mathrm{R}_{b}\right.$-bit rate over code rate), while the electrical filter is modeled as a Gaussian filter of bandwidth $0.65 \mathrm{R}_{b}$. The transmission system considered has a dispersion map composed of SMF section, followed by an EDFA to compensate the fiber losses in SMF section, and DCF section to compensate both GVD and second-order GVD, as well as another EDFA to compensate fiber losses in DCF section.

Using the expression

$$
\text { Uncoded_BER }=(1 / 2) \operatorname{erfc}(Q / \sqrt{2})
$$

the BER of uncoded signal is converted to $Q$ factor in order to make the results comparable with previously reported ones. Coding gain of close to $10 \mathrm{~dB}$ for BER of $10^{-9}$ is found, the result better than any coding scheme proposed so far [3]-[5].
(Since the code rate influence is included in $Q$ factor, the reported coding gain is equivalent to the net effective coding gain of AWGN channel.) To calculate the BER, a bit sequence of length $10^{12}$ is used.

\section{CONCLUSION}

A novel error-control scheme for long-haul optical communication systems based on LDPC codes and iterative decoding has been presented in this letter. The LDPC code is constructed using projective geometries. As opposed to recent papers [3]-[5] where the AWGN assumption is applied, we consider the performance of projective geometry codes in the presence of ASE noise, pulse distortion due to fiber nonlinearities, residual dispersion, crosstalk effects, ISI, etc. The iterative decoding based on normalized min-sum algorithm has been demonstrated to give a coding gain of $10 \mathrm{~dB}$ at BER of $10^{-9}$.

These codes have many unique features such as high code rate, large minimum distances, simple decoding algorithms, encoder can be realized using shift registers, etc., that may allow for very high speed implementations. Moreover, Hagenaruer $e t$ al. [15] realized that the sum-product algorithm is well suited for analog VLSI implementation. This kind of fast, analog iterative decoder is a very attractive option for optical communications.

\section{REFERENCES}

[1] B. Zhu et al., " $3.2 \mathrm{~Tb} / \mathrm{s}(80 \times 42.7 \mathrm{~Gb} / \mathrm{s})$ transmission over $20 \times 100$ $\mathrm{km}$ of nonzero dispersion fiber with simultaneous $\mathrm{C}+\mathrm{L}$-band dispersion compensation," in Proc. Opt. Fiber Commun. Exhibit, 2002, Postdeadline Paper FC8-1-FC8-3.

[2] W. D. Grover, "Forward error correction in dispersion-limited lightwave systems," J. Lightwave Technol., vol. 5/6, pp. 643-654, May 1988.

[3] O. A. Sab, "FEC techniques in submarine transmission systems," in Proc. OFC Conf., vol. 2, 2001, Paper TuF1-3.

[4] O. A. Sab and V. Lemarie, "Block turbo code performances for long-haul DWDM optical transmission systems," in Proc. OFC Conf., vol. 3, 2001, pp. 280-282.

[5] M. Akita et al., "Third generation FEC employing turbo product code for long-haul DWDM transmission systems," in Tech. Dig. Opt. Fiber Commun. Exhibit, 2002, pp. 289-290.

[6] C. Berrou and A. Glavieux, "Near optimum error-correcting coding and decoding: Turbo codes," IEEE Trans. Commun., vol. 44, pp. 1261-1271, Oct. 1996.

[7] B. Vasic, I. B. Djordjevic, and R. Kostuk, "Low-density parity check codes and iterative decoding for long haul optical communication systems," J. Lightwave Technol., to be published.

[8] B. Vasic and I. B. Djordjevic, "Low-density parity check codes for long haul optical communications systems," IEEE Photon. Technol. Lett., vol. 14, pp. 1208-1210, Aug. 2002.

[9] B. Vasic and I. B. Djordjevic, "Iteratively decodable block codes in long-haul optical transmission systems," J. Opt. Commun., vol. 23, no. 5, pp. 182-186, 2002.

[10] Y. Kou, S. Lin, and M. P. C. Fossorier, "Low-density parity-check codes based on finite geometries: A rediscovery and new results," IEEE Trans. Inform. Theory, vol. 47, pp. 2711-2736, July 2001.

[11] G. P. Agrawal, Nonlinear Fiber Optics. San Diego, CA: Academic, 2001.

[12] I. B. Djordjevic and B. Vasic, "An advanced direct detection receiver model," J. Opt. Commun., to be published.

[13] N. S. Bergano and C. R. Davidson, "Circulating loop transmission experiments for the study long-transmission systems using erbium-doped fiber amplifiers," J. Lightwave Technol., vol. 13, pp. 879-888, May 1995.

[14] R. Lucas, M. P. C. Fossorier, Y. Kou, and S. Lin, "Iterative decoding of one-step majority logic decodable codes on belief propagation," IEEE Trans. Commun., vol. 48, pp. 931-937, June 2000.

[15] J. Hagenauer et al., "Analog turbo-networks in VLSI: The next step in turbo decoding and equalization," presented at the 2nd Intl. Symp. Turbo Codes, Brest, France, Sept. 4-7, 2000. 